\title{
Correction to: Stem Cell-Derived Exosomes as Treatment for Stroke: a Systematic Review
}

\author{
Leila Dehghani ${ }^{1}$ (D) Seyed Mahmoud Hashemi ${ }^{1,2} \cdot$ Mohammad Saadatnia $^{3} \cdot$ Alireza Zali $^{4} \cdot$ Saeed Oraee-Yazdani ${ }^{4}$. \\ Saeed Heidari Keshel ${ }^{1}$. Arash Khojasteh ${ }^{5}$ - Masoud Soleimani ${ }^{1,6,7}$
}

Published online: 20 March 2021

(C) Springer Science+Business Media, LLC, part of Springer Nature 2021

\section{Correction to: Stem Cell Reviews and Reports https://doi.org/10.1007/s12015-020-10024-7}

The original version of this article unfortunately contained a mistake. One of the affiliations was incorrect. The correct information is given below.

The original article has been corrected.

Publisher's Note Springer Nature remains neutral with regard to jurisdictional claims in published maps and institutional affiliations.

The online version of the original article can be found at https://doi.org/ $10.1007 / \mathrm{s} 12015-020-10024-7$

Arash Khojasteh

arashkhojasteh@gmail.com; https://www.scopus.com/authid/

detail.uri?authorId=24068894800

Seyed Mahmoud Hashemi

https://www.scopus.com/authid/detail.uri?authorId=27169527500

Mohammad Saadatnia

https://www.scopus.com/authid/detail.uri?authorId=16234564400

Alireza Zali

https://www.scopus.com/authid/detail.uri?authorId=23011000500

Saeed Oraee-Yazdani

https://www.scopus.com/authid/detail.uri?authorId=56646543900

Saeed Heidari Keshel

https://www.scopus.com/authid/detail.uri?authorId=57216905594

Masoud Soleimani

soleim_m@modares.ac.ir; http://www.scopus.com/authid/

detail.url?authorId=57200104836
1 Department of Tissue Engineering and Applied Cell Sciences, School of Advanced Technologies in Medicine, Student Research Committee, Shahid Beheshti University of Medical Sciences, Tehran, Iran

2 Department of Immunology, School of Medicine, Shahid Beheshti University of Medical Sciences, Tehran, Iran

3 Department of Neurology, School of Medicine, Isfahan University of Medical Sciences, Isfahan, Iran

4 Functional Neurosurgery Research Center, Shohada Tajrish Comprehensive Neurosurgical Center of Excellence, Shahid Beheshti University of Medical Sciences, Tehran, Iran

5 Department of Tissue Engineering and Applied Cell Sciences, School of Advanced Technologies in Medicine, Shahid Beheshti University of Medical Sciences, Tehran, Iran

6 Department of Hematology, Faculty of Medical Sciences, Tarbiat Modares University, Tehran, Iran

7 Medical Nanotechnology and Tissue engineering Research Center, Shahid Beheshti University of Medical Sciences, Tehran, Iran 